\title{
Biological functions of lung cancer cells are suppressed in co-culture with mesenchymal stem cells isolated from umbilical cord
}

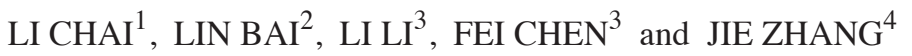 \\ ${ }^{1}$ Core Facility; ${ }^{2}$ Regenerative Medicine Research Centre; ${ }^{3}$ Laboratory of Pathology; \\ ${ }^{4}$ Key Laboratory of Transplant Engineering and Immunology, Ministry of Health, \\ West China Hospital, Sichuan University, Chengdu, Sichuan 610041, P.R. China
}

Received June 16, 2017; Accepted November 3, 2017

DOI: $10.3892 /$ etm.2017.5456

\begin{abstract}
Stem cell-based therapy serves a key role in clinical treatments, and mesenchymal stem cells (MSCs) have been widely used in clinical tumor therapy trials. In the present study, MSCs were isolated from umbilical cord (UC) and co-cultured with the lung cancer cell line H1299. The effects of UC-derived MSCs (UCMSCs) on H1299 cell invasion and proliferation were evaluated using a Matrigel-based Transwell assay and CCK8 assay, respectively. Apoptosis and cell cycle progression among H1299 cells were detected by flow cytometry, and kinase expression in H1299 cells was detected by western blotting. The results indicated that UCMSCs significantly inhibited H1299 cell invasion and significantly induced apoptosis of H1299 cells, but exhibited no effect on H1299 cell proliferation and cell cycle progression. It was also identified that H1299 cell expression of key kinases (AKT, phosphoinositide 3-kinase, signal transducer and activator of transcription 3 and mechanistic target of rapamycin) was significantly suppressed in the presence of UCMSCs. To the best of our knowledge, the present study demonstrates for the first time that UCMSCs have an anti-tumor effect against lung cancer cells, which may indicate that AKT/phosphoinositide 3-kinase/signal transducer and activator of transcription 3 signaling is important in the UCMSC-mediated regulation of H1299 cell functions.
\end{abstract}

\section{Introduction}

Mesenchymal stem cells (MSCs) are a key type of multipotent stem cells in clinical applications (1). MSCs are ubiquitous and can be isolated from bone marrow, skin, heart, adipose tissue,

Correspondence to: Professor Jie Zhang, Key Laboratory of Transplant Engineering and Immunology, Ministry of Health, West China Hospital, Sichuan University, 88 Keyuan South Road, Chengdu, Sichuan 610041, P.R. China

E-mail: zjie@scu.edu.cn

Key words: mesenchymal stem cells, lung cancer, H1299, anti-tumor effect brain, deciduous teeth, umbilical cord (UC) and peripheral blood (2). In specific in vivo microenvironments or when cultured in specific differentiation medium, MSCs can be induced to differentiate into many cell types including adipocytes, tenocytes, osteoblasts and visceral mesoderm (3). MSCs also reduce immune responses by suppressing the activation and proliferation of immune cells such as T cells (4), B cells (5), natural killer cells (6) and antigen-presenting cells, and the complement system (7). Regarding surface marker expression, MSCs are negative for co-stimulatory molecules CD80, CD86 and human leukocyte antigen-II and positive for CD29, CD90 and CD59, which makes MSCs unable to stimulate allo- or xenogeneic lymphocytes due to a lack of immunogenicity (8). MSCs also secrete multiple trophic molecules that can benefit remote tissues and serve a key function in tissue repair (9).

Based on cellular characteristics, MSCs are now the best candidate in many cell-based therapies. Their immunosuppressive capability serves a key function in the induction of transplantation tolerance and protects solid organ grafts from rejection (10). In autoimmune disease treatment, engraftment of MSCs improves the levels of serological markers and stabilizes renal function by preventing the appearance of serious adverse events (11). MSCs have also been widely used in many treatment trials, including stem cell-based therapies for liver cirrhosis (12), cerebral palsy (13), type I diabetes (14), multiple sclerosis (15) and graft-vs.-host disease (16). However, the effect of MSCs on lung cancer cells remains unclear. In the present study, UC-derived MSCs (UCMSCs) were co-cultured with H1299 lung cancer cells in order to evaluate how MSCs influence the biological functions of H1299 cells.

\section{Materials and methods}

Cells and co-culture system. H1299 cells (State key Laboratory of Biological Treatment, Sichuan University; Chengdu, China) were maintained in RPMI-1640 (Gibco; Thermo Fisher Scientific, Inc., Waltham, MA, USA) with $10 \%$ fetal bovine serum (FBS; Invitrogen; Thermo Fisher Scientific, Inc.) and in a sterile humidified incubator with $5 \% \mathrm{CO}_{2}$ at $37^{\circ} \mathrm{C}$. UCMSCs were obtained from the Sichuan Umbilical Cord Blood Stem Cell Bank (Chengdu, China). After dissociation 
in a $37^{\circ} \mathrm{C}$ water bath, UCMSCs were cultured in Dulbecco's modified Eagle's medium (DMEM; Invitrogen; Thermo Fisher Scientific, Inc.) with 10\% FBS (Invitrogen; Thermo Fisher Scientific, Inc.) at $1 \times 10^{5}$ cells/well in 6 -well plates, and all cells were grown in a sterile humidified incubator with $5 \% \mathrm{CO}_{2}$ at $37^{\circ} \mathrm{C}$. The medium was changed every 2 days, and adherent cells were harvested after 2 weeks by $0.25 \%$ trypsin (Gibco; Thermo Fisher Scientific, Inc.) treatment. Only UCMSCs from passages 6 or lower were used for co-culture. UCMSCs were co-cultured with H1299 cells in DMEM with 10\% FBS and in a sterile humidified incubator with $5 \% \mathrm{CO}_{2}$ at $37^{\circ} \mathrm{C}$ for 24,48 , 72 and $96 \mathrm{~h}$. The co-culture ratio of UCMSCs to H1299 cells was 2:1. H1299 cells were considered the control group and underwent the same culturing time (24, 48, 72 and $96 \mathrm{~h})$.

Transwell-based invasion assay. An invasion assay was conducted using 24-well (8- $\mu \mathrm{m}$ pore size) Transwell plates (Corning, Inc., Corning, NY, USA). H1299 cells were plated in the upper chambers in DMEM at $3 \times 10^{4}$ cells/well, which were pre-coated with $20 \%$ Matrigel (BD Biosciences, Franklin Lakes, NJ, USA), while UCMSCs were maintained in the bottom chamber in DMEM with 1\% FBS. After 24 or 48 h, invading H1299 cells were detected by crystal violet staining at room temperature for $30 \mathrm{~min}$ (which was synchronous with the culturing time) and all the results were observed using light microscopy (magnification, x100).

Assays for proliferation, apoptosis and cell cycle analysis. CCK-8 detection (Cell Counting Kit-8; Dojindo Molecular Technologies, Inc., Kumamoto, Japan) was performed to assess H1299 cell proliferation at 24,48 and $72 \mathrm{~h}$ in co-culture. At 24, 48 and $72 \mathrm{~h}$ in co-culture, apoptosis was evaluated by staining of $\mathrm{H} 1299$ cell cultures with $3 \mu \mathrm{l}$ Annexin V (fluorescein isothiocyanate) at room temperature for $20 \mathrm{~min}$, followed by counterstaining with $5 \mu \mathrm{l}$ propidium iodide at room temperature for $5 \mathrm{~min}$ and detection by flow cytometry. Cell cycle progression among H1299 cells was also investigated by flow cytometry.

Western blot analysis. Co-cultured H1299 cells were collected by two washes with cold PBS, and proteins were extracted using radioimmunoprecipitation assay protein lysis reagent (Pierce; Thermo Fisher Scientific, Inc.) containing $1 \mathrm{X}$ protease inhibitors (Roche Diagnostics, Indianapolis, IN, USA). The protein concentration was measured using a Micro BCA Protein Assay kit (Pierce; Thermo Fisher Scientific, Inc.). Sodium dodecyl sulfate-polyacrylamide gel (12\%) electrophoresis was used to separate proteins (30 $\mu \mathrm{g}$ per lane), which were then transferred onto nitrocellulose membranes (Invitrogen; Thermo Fisher Scientific, Inc.). After blocking with $5 \%$ fat-free milk at room temperature for $1 \mathrm{~h}$, membranes were incubated with primary antibodies at $4^{\circ} \mathrm{C}$ overnight and then horseradish peroxidase-conjugated secondary antibody at room temperature for $2 \mathrm{~h}$ (1:5,000; cat. no. ab6789\&ab6721; Abcam, Cambridge, UK). Antigen-antibody complexes were visualized using an enhanced chemiluminescence reagent (GE Healthcare, Chicago, IL, USA). Primary antibodies were as follows: AKT (1:800; cat. no. AF6261), p-AKT (1:800; cat. no. AF0016), phosphoinositide 3-kinase (PI3K; 1:800; cat. no. AF6242), p-PI3K (1:800; cat. no. AF3241), signal transducer and activator of transcription 3 (STAT3; 1:1,000; cat. no. AF6294), p-STAT3 (1:1,000; cat. no. AF3294), extracellular signal-regulated kinase 1/2 (ERK1/2; 1:1,000; cat. no. AF0155), p-ERK1/2 (1:1,000; cat. no. AF1015), mechanistic target of rapamycin (mTOR; 1:800; cat. no. AF6308), p-mTOR (1:800; cat. no. AF3310) (all Affinity Biosciences, Zhenjiang, China) and GAPDH (1:2,000; cat. no. 200608; Zen BioScience Co., Ltd., Chengdu, China).

Data analysis. Image Lab software 5.1 (Bio-Rad Laboratories, Inc., Hercules, CA, USA) and ModFit LT version 4.1 (Verity Software House, Topsham, ME, USA) were used for analysis of western blotting and cell cycle data, respectively. All analyzed data are expressed as the mean \pm standard error, as calculated using SPSS 19.0 (IBM Corp, Armonk, NY, USA). T-tests were performed to evaluate inter-group differences. $\mathrm{P}<0.05$ was considered to indicate a statistically significant difference. All figures were generated using GraphPad Prism 5 (GraphPad Software, Inc., La Jolla, CA, USA).

\section{Results}

UCMSCs significantly suppressed invasion of H1299 cells. The invasion ability of H1299 cells in the presence of UCMSCs was detected in Transwell chambers coated with Matrigel. As shown in Fig. 1, the number of invading H1299 cells was significantly reduced in the co-culture group compared with the control (H1299 cells only) group following 24 and $48 \mathrm{~h}$ in culture $(\mathrm{P}<0.001)$. This indicated that UCMSCs could inhibit the invasion of H1299 cells.

UCMSCs induced apoptosis of H1299 cells. Flow cytometry was conducted to identify changes in the apoptosis rate of H1299 cells in co-culture with UCMSCs according to Annexin $\mathrm{V}$ staining. The results indicated that the apoptosis rates of H1299 cells did not differ markedly between the control and co-culture groups at either $48 \mathrm{~h}(3.0$ vs. $2.7 \%)$ or $72 \mathrm{~h}(3.1$ vs. $6.8 \%$; Fig. 2). However, after $96 \mathrm{~h}$ in co-culture, the apoptosis rate of H1299 cells in the co-culture group (57.4\%) was greater than that among $\mathrm{H} 1299$ cells cultured alone (2.4\%).

UCMSCs inhibited H1299 cell proliferation but exhibited no effect on cell cycle progression. The cell cycle distribution of H1299 cells was detected by flow cytometry. The results indicated no differences between H1299 cells cultured alone and with UCMSCs for either 48 h (control cells: G0/G1 62.42\%, G2/M 10.32\%, S 27.26\%; co-cultured cells: G0/G1 63.95\%, G2/M 7.92\%, S 28.13\%) or 72 h (control cells: G0/G1 61.05\%, G2/M 15.62\%, S 23.33\%; co-cultured cells: G0/G1 61.42\%, G2/M 9.13\%, S 29.45\%; Fig. 3A and B). The proliferation of H1299 cells was then evaluated by CCK8 assay after 24, 48 or $72 \mathrm{~h}$ in co-culture with UCMSCs. The results indicated that H1299 cell proliferation was not significantly influenced by UCMSCs in co-culture, although a slight increase after 24, $48 \mathrm{~h}$ and a slight decrease after $72 \mathrm{~h}$ were observed.

UCMSCs inhibited PI3K/AKT kinase expression in $H 1299$ cells. Previous research has demonstrated that the PI3K/AKT/STAT3 pathway serves a key function in mediating the occurrence of tumor metastasis (17). In the present study, the expression of these kinases was detected by western blotting 

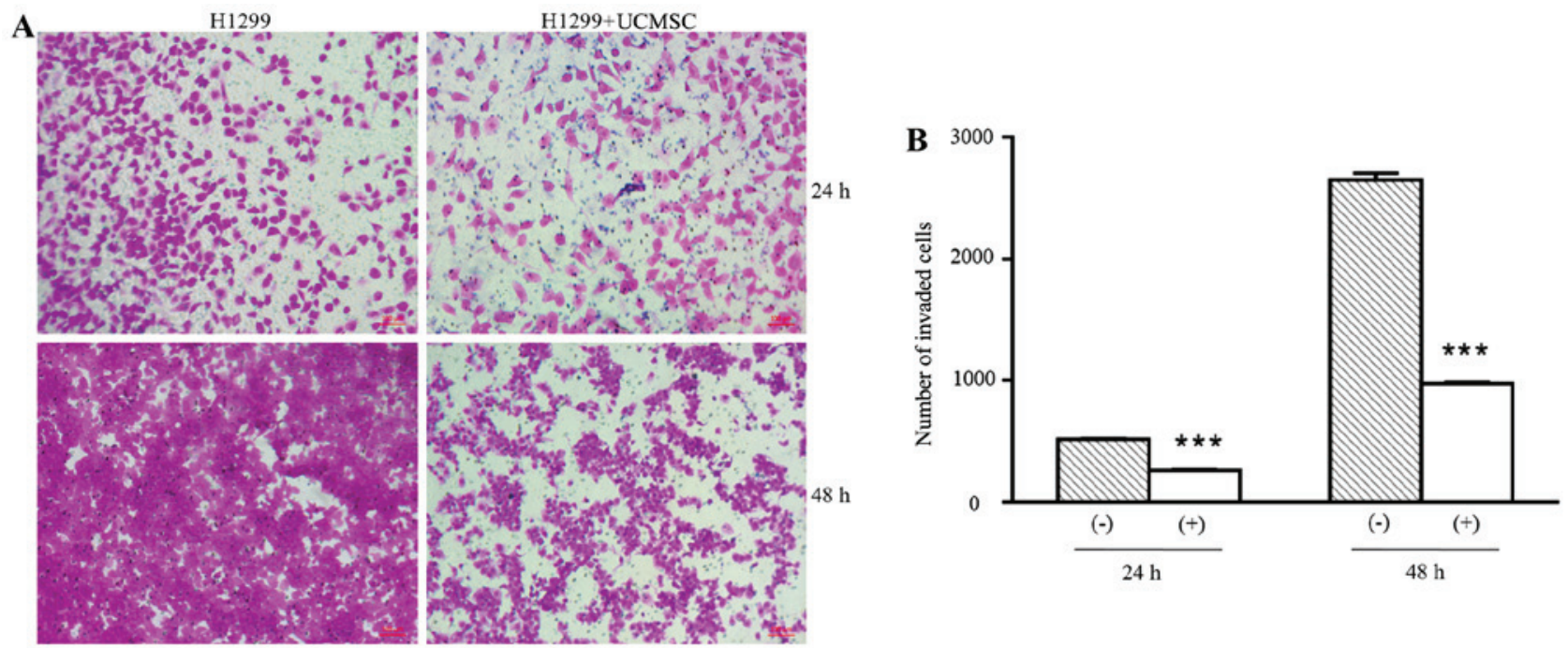

Figure 1. Invasion of H1coo299 cells co-cultured with UCMSCs. (A) Micrographs of H1299 cells that migrated through Transwell membranes coated with Matrigel. (B) Quantitative analysis of $\mathrm{H} 1299$ cell invasion. Scale bar $=100 \mu \mathrm{m} .{ }^{* * *} \mathrm{P}<0.001$ vs. control cells. UCMSC, umbilical cord-derived mesenchymal stem cell; (-), control cells; (+), co-cultured cells.

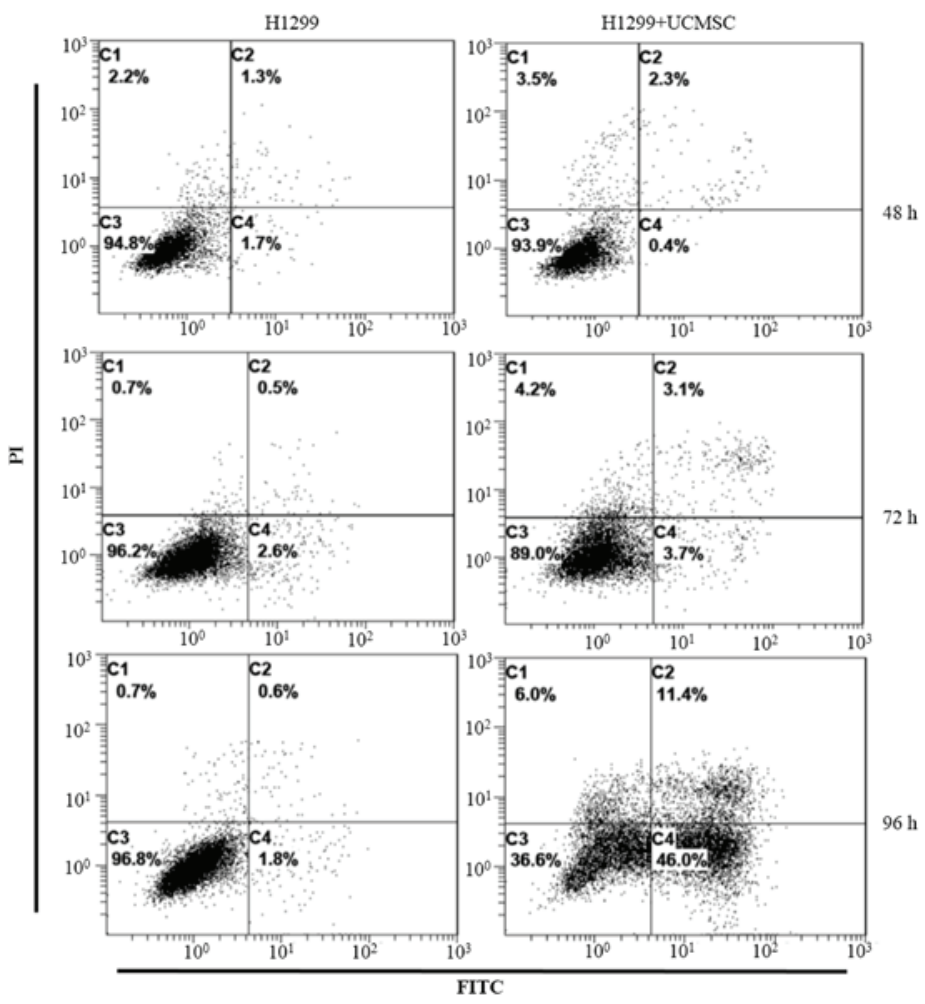

Figure 2. Flow cytometric detection of apoptotic H1299 cells in co-culture with umbilical cord-derived mesenchymal stem cells. PI, propidium iodide; FITC, fluorescein isothiocyanate.

in order to explore the mechanisms by which UCMSCs inhibit the invasive ability of H1299 cells (Fig. 4). Expression of AKT in H1299 cells exhibited no change after $48 \mathrm{~h}$ in co-culture with UCMSCs compared with the control cells. However, the expression of AKT was significantly inhibited at $72 \mathrm{~h}(\mathrm{P}<0.05)$. p-AKT expression was significantly inhibited in co-cultured H1299 cells compared with control cells at $48(\mathrm{P}<0.05)$ and $72 \mathrm{~h}(\mathrm{P}<0.01)$. PI3K and p-PI3K expression levels were also significantly reduced after 48 (PI3K, $\mathrm{P}<0.001$; $\mathrm{p}-\mathrm{PI} 3 \mathrm{~K}, \mathrm{P}<0.05)$ and $72 \mathrm{~h}(\mathrm{P}<0.001)$ in co-culture compared with control cells. Co-culture with UCMSCs resulted in inhibited expression of STAT3 $(\mathrm{P}<0.001)$ and $\mathrm{p}-\mathrm{STAT} 3(\mathrm{P}<0.05)$ in H1299 cells only after $72 \mathrm{~h}$ in co-culture. ERK1/2 expression was significantly decreased after $72 \mathrm{~h}(\mathrm{P}<0.001)$. $\mathrm{p}$-ERK expression was significantly increased after $48 \mathrm{~h}(\mathrm{P}<0.001)$ and then significantly inhibited after $72 \mathrm{~h}(\mathrm{P}<0.01)$ in co-culture compared with control cells. Finally, after 48 and $72 \mathrm{~h}$ in co-culture with UCMSCs, expression of mTOR $(\mathrm{P}<0.01)$ and p-mTOR $(48 \mathrm{~h}$, 

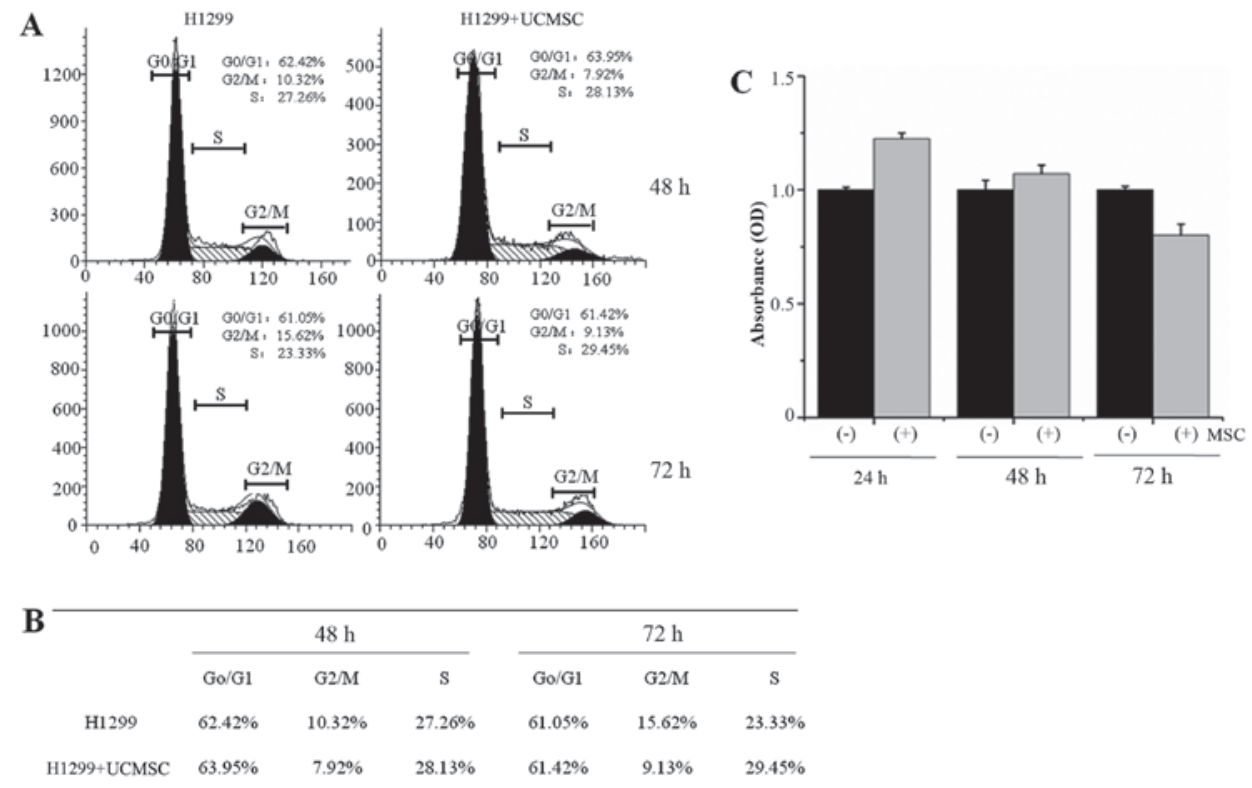

Figure 3. Cell cycle distribution and proliferation of H1299 cells in co-culture with UCMSCs. (A) Flow cytometric detection of H1299 cells in each phase of the cell cycle. (B) Quantitative analysis of cell cycle distribution. (C) Proliferation of H1299 cells as detected by CCK8 assay. UCMSC, umbilical cord-derived mesenchymal stem cell; OD, optical density; (-), control cells; (+), co-cultured cells.

A

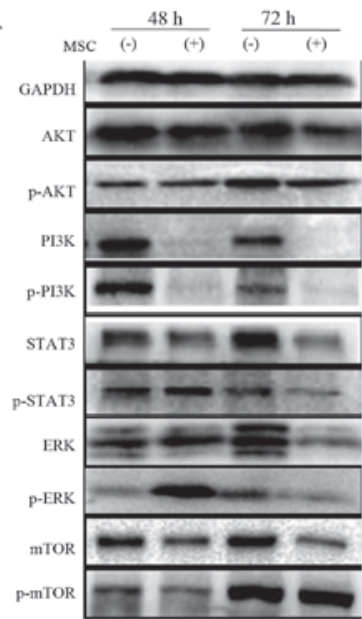

B
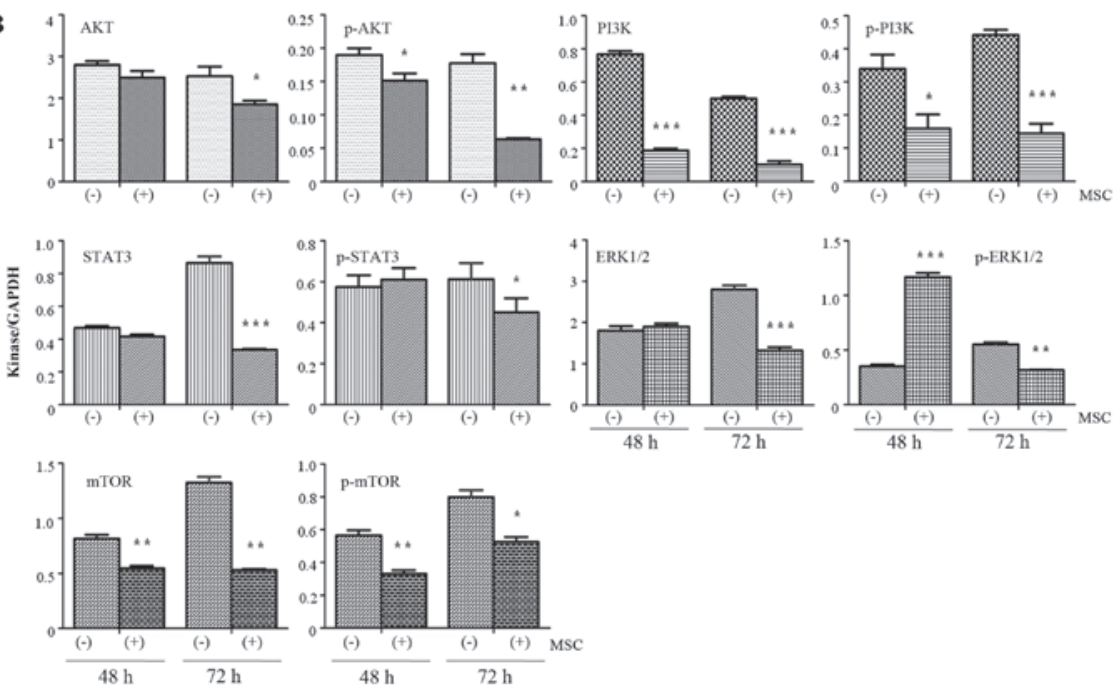

Figure 4. Kinase expression in H1299 cells co-cultured with umbilical cord-derived mesenchymal stem cells. (A) Western blot analysis results. (B) Quantitative analysis of kinase expression. ${ }^{*} \mathrm{P}<0.05,{ }^{* *} \mathrm{P}<0.01,{ }^{* * * *} \mathrm{P}<0.001$ vs. control cells. PI3K, phosphoinositide 3-kinase; STAT3, signal transducer and activator of transcription 3; ERK1/2, extracellular signal-regulated kinase 1/2; mTOR, mechanistic target of rapamycin; (-), control cells; (+), co-cultured cells. 
$\mathrm{P}<0.01 ; 72 \mathrm{~h}, \mathrm{P}<0.05$ ) was significantly inhibited. These observations indicated that the AKT/PI3K/STAT3/mTOR pathways are involved in the effects of USMSCs on the biological functions of H1299 cells.

\section{Discussion}

To the best of our knowledge, the effect of UCMSCs on the biological functions of lung cancer cells has not previously been reported. In the present study, H1299 cells were co-cultured with UCMSCs in order to investigate how UCMSCs influenced the biological functions of H1299 cells. This was analyzed using numerous detection methods, including CCK8 assay (proliferation), flow cytometry (apoptosis and cell cycle), Transwell Matrigel assay (invasion) and western blot analysis (expression of kinases). The results indicated that UCMSCs inhibited invasion and induced apoptosis of H1299 cells, but exerted little influence on H1299 cell cycle distribution or proliferation. Further analyses suggested that expression of multiple kinases, including AKT, PI3K, ERK, STAT3 and mTOR, in either phosphorylated or non-phosphorylated states, was significantly suppressed in H1299 cells co-cultured with UCMSCs. Thus, the present study indicated that UCMSCs could inhibit the biological functions of H1299 cells by suppressing activation of AKT/PI3K/STAT3/mTOR signaling.

Experimental evidence on the effects of MSCs on tumor cells remains contradictory. Consistent with the present results, Wu et al (18) demonstrated that microvesicles from human UCMSCs could inhibit the functions of T24 bladder cancer cells. The study also demonstrated that this proliferation suppression was mediated by cell cycle arrest and the induction of apoptosis was mediated by increased expression of caspase 3. In a study of the effects of MSCs on cholangiocarcinoma, Liu et al (19) established xenograft models by injection of HCCC-9810 cells and identified that conditioned medium from UCMSCs inhibited proliferation and induced apoptosis in a dose- and time-dependent manner. In another study, $\alpha$-smooth muscle actin-positive MSCs were co-engrafted with pancreatic cancer cells in severe combined immunodeficiency mice and the results confirmed that MSCs could promote epithelial-mesenchymal transition (20). Mechanistic analysis indicated that the 'stemness' of pancreatic cells was enhanced by Notch-associated signaling regulated by MSCs (20). Zhang et al (21) co-cultured breast cancer and prostate cancer cells with bone marrow-derived MSCs and identified that treatment with MSCs significantly enhanced angiogenesis within the tumor in nude mice. Considering all these findings together, the effects of MSCs on the biological functions of cancer cells remain controversial. Possible explanations for this include heterogeneity of MSCs or variations in microenvironments.

To the best of our knowledge, the present study was the first to demonstrate that UCMSCs could suppress lung cancer cell functions by inhibiting invasion and inducing apoptosis. In the present mechanistic analysis, it was identified that expression of multiple key kinases (AKT, PI3K, STAT3 and mTOR) by H1299 cells was inhibited in the presence of UCMSCs. This may indicate that AKT/PI3K/STAT3 signaling is important in the UCMSC-mediated regulation of H1299 cell functions. Further studies are required to confirm this hypothesis.

\section{References}

1. Deans RJ and Moseley AB: Mesenchymal stem cells: Biology and potential clinical uses. Exp Hematol 28: 875-884, 2000.

2. Pittenger MF, Mackay AM, Beck SC, Jaiswal RK, Douglas R, Mosca JD, Moorman MA, Simonetti DW, Craig S and Marshak DR: Multilineage potential of adult human mesenchymal stem cells. Science 284: 143-147, 1999.

3. Phinney DG and Prockop DJ: Concise review: Mesenchymal stem/multipotent stromal cells: The state of transdifferentiation and modes of tissue repair-current views. Stem Cells 25: 2896-2902, 2007.

4. Glennie S, Soeiro I, Dyson PJ, Lam EW and Dazzi F: Bone marrow mesenchymal stem cells induce division arrest anergy of activated T cell. Blood 105: 2821-2827, 2005.

5. Ren G, Su J, Zhang L, Zhao X, Ling W, L'huillie A, Zhang J, $\mathrm{Lu}$ Y, Roberts AI, Ji W, et al: Species variation in the mechanisms of mesenchymal stem cell-mediated immunosuppression. Stem Cells 27: 1954-1962, 2009.

6. Sotiropoulou PA, Perez SA, Gritzapis AD, Baxevanis CN and Papamichail M: Interactions between human mesenchymal stem cells and natural killer cells. Stem Cells 24: 74-85, 2006.

7. Jiang XX, Zhang Y, Liu B, Zhang SX, Wu Y, Yu XD and Mao N: Human mesenchymal stem cells inhibit differentiation and function of monocyte-derived dendritic cells. Blood 105: 4120-4126, 2005.

8. Gronthos S, Franklin DM, Leddy HA, Robey PG, Storms RW and Gimble JM: Surface protein characterization of human adipose tissue derived stromal cells. J Cell Physiol 189: 54-63, 2001.

9. Bassi E, Aita CA and Câmara NO: Immune regulatory properties of multipotent mesenchymal stromal cells: Where do we stand? World J Stem Cell 3: 1-8, 2011.

10. Rasmusson I: Immune modulation by mesenchymal stem cells. Exp Cell Res 312: 2169-2179, 2006.

11. Herzog EL, Chai L and Krause DS: Plasticity of marrow-derived stem cells. Blood 102: 3483-3493, 2003.

12. Kharaziha P, Hellström PM, Noorinayer B, Farzaneh F, Aghajani K, Jafari F, Telkabadi M, Atashi A, Honardoost M, Zali MR and Soleimani M: Improvement of liver function in liver cirrhosis patients after autologous mesenchymal stem cell injection: A phase I-II clinical trial. Eur J Gastroenterol Hepatol 21: 1199-1205, 2009.

13. Zhang Z, Lin H, Shi M, Xu R, Fu J, Lv J, Chen L, Lv S, Li Y, Yu S, et al: Human umbilical cord mesenchymal stem cells improve liver function and ascites in decompensated liver cirrhosis patients. J Gastroenterol Hepatol 2 (Suppl 27): S112-S120, 2012.

14. Vojtassák J, Danisovic L, Kubes M, Bakos D, Jarábek L, Ulicná $\mathrm{M}$ and Blasko M: Autologous biograft and mesenchymal stem cells in treatment of the diabetic foot. Neuro Endocrinol Lett 2 (Suppl 27): S134-S137, 2006.

15. Djouad F, Plence P, Bony C, Tropel P, Apparailly F, Sany J, Noël D and Jorgensen C: Immunosuppressive effect of mesenchymal stem cells favors tumor growth in allogeneic animals. Blood 102: 3837-3844, 2003.

16. Le Blanc K, Frassoni F, Ball L, Locatelli F, Roelofs H, Lewis I, Lanino E, Sundberg B, Bernardo ME, Remberger M, et al: Mesenchymal stem cells for treatment of steroid-resistant, severe, acute graft-versus-host disease: A phase II study. Lancet 371: 1579-1586, 2008

17. Valastyan S and Weinberg RA: Tumor metastasis: Molecular insights and evolving paradigms. Cell 147: 275-292, 2011.

18. Wu S, Ju GQ, Du T, Zhu YJ and Liu GH: Mcirovesicles derived from human umbilical cord Wharton's jelly mesenchymal stem cells attenuate bladder tumor cell growth in vitro and in vivo. PLoS One 8: e61366, 2013.

19. Liu J, Han G, Liu H and Qin C: Suppression of cholangiocarcinoma cell growth by human umbilical cord mesenchyaml stem cells: A possible role of wnt and Akt signaling. PLoS One 8: e62844, 2013.

20. Kabashima-Niibe A, Higuchi H, Takaishi H, Masugi Y, Matsuzaki Y, Mabuchi Y, Funakoshi S, Adachi M, Hamamoto Y, Kawachi S, et al: Mesenchymal stem cells regulate epithelial-mesenchymal transition and tumor progression of pancreatic cancer cells. Cancer Sci 104: 157-164, 2013.

21. Zhang T, Lee YW, Rui YF, Cheng TY, Jiang XH and Li G: Bone marrow-derived mesenchymal stem cells promote growth and angiogenesis of breast and prostate tumors. Stem Cell Res Ther 4: 70, 2013 